\title{
CHA2DS2-VASc score can guide the screening of atrial fibrillation - cross-sectional study in a geriatric ward
}

This article was published in the following Dove Press journal: Clinical Interventions in Aging

Zyta Beata Wojszel (D) ${ }^{1,2}$

Agnieszka Kasiukiewicz ${ }^{1,2}$

Marta Swietek ${ }^{2,3}$

Michal Lukasz Swietek ${ }^{3}$ Lukasz Magnuszewski ${ }^{2,3}$

'Department of Geriatrics, Medical University of Bialystok, Bialystok, Poland; ${ }^{2}$ Department of Geriatrics, Hospital of the Ministry of Interior in Bialystok, Bialystok, Poland; ${ }^{3}$ Doctoral Studies, Department of Geriatrics, Medical University of Bialystok, Bialystok, Poland
Purpose: Atrial fibrillation (AF) is an increasingly common rhythm disorder and an important risk factor of ischemic stroke, heart failure, hospitalization, and cardiovascular mortality. Its diagnosis, however, is often delayed because of silent character of the arrhythmia. The aim of the study was to identify independent determinants of AF in patients of the geriatric ward, so as to be able to propose a strategy for screening of this arrhythmia.

Methods: Cross-sectional cohort study of patients admitted to the department of geriatrics was conducted. The prevalence of AF and its health correlates (including AF recognized risk factors) was assessed. Relative risks were calculated and multivariable logistic regression analysis model was built. The predictive performance was evaluated using receiver-operating characteristic (ROC) curve analysis.

Results: There were 416 patients hospitalized in the study period and 98 (23.6\%) presented with AF. The independent predictors with top 3 strongest association with $\mathrm{AF}$ were congestive heart failure (OR 5.43; 95\%CI 3.14-9.39; $P<0.001$ ), age of 75+years (OR 4.0; 95\% CI 1.43-11.2; $P=0.008$ ), and previous history of stroke or transient ischemic attack (OR 2.1; 95\% CI 1.06-4.13; $P=0.03$ ). ROC analysis showed CHA2DS2-VASc scale significance as a screening tool for AF (ROC-AUC 0.75; $0.7-0.8 ; P<0.001$ ), with the value of 4 or more as the best cut-off point.

Conclusions: Based on CHA2DS2-VASc score the intensity of surveillance for AF at a primary prevention population level could be probably guided, but it requires further research.

Keywords: atrial fibrillation screening, older people, risk factors and determinants, CHA2DS2-VASc score

\section{Plain language summary}

- Atrial fibrillation (AF) is the most frequent cardiac arrhythmia in old age, connected with the high risk of thromboembolic complications. Up to $40 \%$ of its cases are still undetected, without anticoagulant prophylaxis.

- More sensitive and specific screening strategies aimed at detecting AF for geriatric patients in primary care could allow to better target the health care system resources for screening purposes in this highly dependent and frail patients.

- The aim of the study was to identify the prevalence of AF and its determinants in patients hospitalized in a geriatric ward.

Correspondence: Zyta Beata Wojsze Department of Geriatrics, Medical University in Bialystok, Fabryczna str. 27,

15-47| Bialystok, Poland

$\mathrm{Tel}+48858694982$

Email wojszel@umb.edu.pl
- We showed that congestive heart failure, history of stroke or transient ischemic attack, and age 75 years or above are the main independent predictors of AF in patients hospitalized in the geriatric ward. 
- We confirmed that the CHA2DS2-VASc scale can be a first-step screening instrument. It allowed to discriminate between individuals with and without $\mathrm{AF}$, with the value 4 or more as the best cut-off point.

\section{Introduction}

Atrial fibrillation (AF) is one of the most common rhythm disorders. Its prevalence in general adult population ranges between $1.5 \%$ and $2 \%{ }^{1}$ however, it increases with age, and patients over 65 years old represent more than three-quarters of AF cases. ${ }^{2} \mathrm{AF}$ is connected with high thromboembolic risk and thromboembolic events complications, ${ }^{3}$ increases the risk of heart failure and cardiovascular mortality, ${ }^{4}$ leads to lower quality of life, greater activity impairment, increased odds of hospitalization, ${ }^{5}$ cognitive decline, and mood disorders. ${ }^{6,7}$

The early diagnosis and anticoagulant treatment are crucial to limit the negative consequences of the arrhythmia, but a large proportion of older patients are not treated with oral anticoagulant medications. ${ }^{8}$ Therefore, recent European Society of Cardiology (ESC) Guidelines on management of AF highlight the role of early diagnosis of arrhythmia, which is often clinically silent. ${ }^{9}$ Opportunistic screening for silent AF by pulse taking or ECG in patients over 65 years of age during routine medical visits is recommended. Moreover, systematic ECG screening of all patients over 75 years old, or at high risk of stroke, may be considered. This is often difficult to implement, as older patients in advanced age are often functionally impaired and not able to visit doctor's office. ${ }^{10}$

Different approaches for AF screening are applied, using new, quite simple methods, like single-lead ECG, modified sphygmomanometers, finger-probe devices, and new organizational solutions. They can potentially enhance the detection of AF and overcome certain limitations of the conventional methods. ${ }^{11,12}$ However up to $40 \%$ of AF cases are still undetected. $^{13}$ Therefore, better actions targeted on screening AF should be sought, that would allow more effective exploitation of often poor health care system resources. It is important to indicate the most significant arrhythmia predictive factors. Screening should be directed primarily to this group. More sensitive and specific screening strategies for geriatric patients in primary care, aimed at detecting $\mathrm{AF}$, could allow to better target the health care system resources for screening purposes in this highly dependent and frail group.
The aim of the study was to identify the prevalence of $\mathrm{AF}$ and its determinants in patients hospitalized in the geriatric ward.

\section{Material and methods}

Study design and setting

We developed the prospective cross-sectional study on older patients hospitalized in the Department of Geriatrics of the Hospital of the Ministry of Interior in Bialystok, Poland, primarily planned as the study on frailty, disability, and multimorbidity in geriatric inpatients. All consecutive patients admitted to the department for the first time between 1 September, 2014 and 30 April, 2015 were enrolled to the evaluation. The additional retrospective analysis of patients' medical records was performed.

The geriatric department is a sub-acute care ward, where older people with multimorbidity and physical and/or cognitive disability are admitted mainly in a planned manner. The average waiting time for admission to the ward is approximately 3 months and the mean length of stay is 7 days. A comprehensive geriatric assessment by the multidisciplinary team is performed during the patients' hospitalization. The above is intended to reduce polytherapy, to identify reasons of patients' functional decline, to diagnose their weight loss, recurrent falls or other geriatric syndromes they are suffered from, which usually - as they are defined as - are multifactorial and often co-exist with each other. Because of that identification of one single major illness as a reason for admission to the ward was not possible in the vast majority of cases.

Patients were interviewed using a structured questionnaire and following data were collected: the age, gender, form and place of residence, history of hospitalization in the last 12 months, comorbidities (of 14 chronic diseases: peripheral arterial disease, ischemic heart disease, chronic cardiac failure (CHF), myocardial infarction (MI), hypertension (HT), stroke, chronic obstructive pulmonary disease, diabetes/prediabetes, neoplasm, dementia, parkinsonism, chronic arthritis, osteoporosis, chronic renal disease), number and type of medications taken before hospitalization. And only those chronic conditions that were confirmed in some way, or newly detected during the current hospitalization, were included in the analysis. Information obtained from the patient was verified by an interview with his or her carer, by a thorough clinical examination, and by a review of all of the patient's 
medical records available. Therefore, it was not a clinical trial in which each participant had completed all the tests included in the research protocol, but a study based on medical data collected in everyday clinical practice. For example, in CHF, echocardiography was performed only if CHF was suspected in a patient without such a diagnosis in earlier medical records or worsening of a previously diagnosed disease. Due to the often limited information on the type and severity of heart failure in the analyzed medical records, we did not specify that in the research. So the variable "CHF" includes both types of this disease (with preserved or reduced ejection fraction) and all (II to IV) NYHA categories of CHF. Multimorbidity was defined as 5 or more diseases of 14 listed above. Polypharmacy was defined as 5 or more drugs taken. The ability to perform basic activities of daily life was assessed with the Barthel Index, ${ }^{14}$ instrumental activities of daily living with Duke OARS I-ADL. ${ }^{15}$ For self-reported level of physical activity, we used the 4-level Saltin-Grimby Physical Activity Level Scale (SGPALS). ${ }^{16}$ Patients were classified as physically inactive (1st level of SGPALS), if during leisure-time they were mainly reading, watching television, using computers or doing other sedentary activities. Body mass index (BMI) was used to assess nutritional health, and patients with $\mathrm{BMI} \geq 30 \mathrm{~kg} / \mathrm{m} 2$ were classified as obese. Anemia was diagnosed if the hemoglobin level was below $8.69 \mathrm{mmol} / \mathrm{L}$ in men and below $7.45 \mathrm{mmol} / \mathrm{L}$ in women. CHA2DS2-VASc and HASBLED scores were calculated. HAS-BLED score $\geq 3$ was interpreted as the high risk of bleeding.

\section{Statistical analysis}

Data were collected and analyzed using IBM SPSS Version 18 Software suit (SPSS, Chicago, IL, USA) and STATISTICA 13.3 software package (TIBCO Software, Palo Alto, CA, USA), and presented as means and standard deviation for normally distributed and as medians and interquartile range for not normally distributed continuous variables, and the number of cases and percentage for categorical variables. Proportions were compared using $\chi 2$ tests, while Student's $t$-test for the independent samples and Mann-Whitney $U$ test were used to compare measures of central tendency (means and medians). Relative risks $(R R \mathrm{~s})$ were calculated to evaluate the potential risk factors that might influence the prevalence of AF in geriatric inpatients. It was followed by a multivariable logistic regression including all predictors with a $P$-value of $R R$ less than 0.1 . The predictive performance of identified significant variables was evaluated using receiver-operating characteristic (ROC) curve analysis. In addition, the best cut-off to maximize sensitivity and specificity was calculated with its confidence interval (CI). A $P$-value of less than 0.05 was regarded as significant. Missing values were omitted and statistics in such cases were calculated for the adequately reduced groups.

The study was approved by the Ethics Committee at Medical University of Bialystok (R-I-002/305/2013). All procedures performed in the study were in accordance with the ethical standards of the Medical University of Bialystok research committee and with the Helsinki declaration and its later amendments. Personal data were not identifiable during the analysis. Written informed consent was obtained from all patients enrolled in the study.

\section{Results}

A total of 416 patients aged $60+$ years were enrolled into the study. AF was diagnosed in 98 patients $(23.6 \%$ of study population). The characteristics of the study groups - with and without AF - are presented in Table 1. The median age of AF participants (AF+ group) was 84 (IQR- 84-87) years and non-AF participants (AF- group) - 82 (IQR-76-86) years, $P=0.002$. Men constituted significantly higher percentage of $\mathrm{AF}$ group comparing to the non-AF group (31.6\% versus $19.8 \% ; P=0.01$ ).

The percentage of patients reporting hospitalization in the last 12 months was significantly higher in the AF group. The number of chronic diseases in the whole population in our research was relatively high, with the median value of 4 (IQR-3-6), and it was significantly higher in the group with AF (Me-6, IQR-5-7 versus Me-4, IQR-3-5 in the control group, $P<0.001$ ). Multimorbidity was diagnosed in $87.8 \%$ of patients with $\mathrm{AF}$ and in $48.1 \%$ of the control group $(P<0.001)$. Parallel to that a positive correlation was found between the presence of $\mathrm{AF}$ and polypharmacy. The number of drugs taken at admittance was high in both groups (Me 7, IQR-5-9), but statistically greater in those with AF (Me 8, IQR-6-10, $P=0.004$ ). Patients with AF significantly more often received antiarrhythmic, anticoagulants, and diuretics, especially loop diuretics and aldosteronereceptor antagonists. Anticoagulants were used solely by $58.2 \%$ of patients with $\mathrm{AF}$, and antiplatelet medications by $26 \%$ of AF patients. The AF group was characterized also by significantly lower GFR values in comparison to the group without AF. The mean value of BMI in patients with $\mathrm{AF}$ rated $30.19 \pm 5.53 \mathrm{~kg} / \mathrm{m}^{2}$ and was similar to mean 
Table I Characteristics of the study groups - with AF (AF+ group) and without AF (AF- group)

\begin{tabular}{|c|c|c|c|c|c|}
\hline Parameter & Total & AF+ group & AF- group & $P$-values ${ }^{a}$ & $\begin{array}{l}\text { Missing } \\
\text { data }\end{array}$ \\
\hline No. (\%) of patients & $416(100.0)$ & $98(23.6)$ & $318(76.4)$ & & \\
\hline Age, y, Me (IQR) & $82(77-86)$ & $84(84.0-87.0)$ & $82(76-86)$ & 0.002 & - \\
\hline Gender, men, n (\%) & 94 (22.09) & $31(31.6)$ & $63(19.8)$ & 0.01 & - \\
\hline Place of residence, rural, $\mathrm{n}(\%)$ & $87(20.9)$ & $21(21.4)$ & $66(20.8)$ & 0.89 & - \\
\hline Education, elementary or less, n (\%) & $231(66.2)$ & $54(65.9)$ & $177(65.3)$ & 0.92 & 65 \\
\hline Living alone, n (\%) & $119(29,8)$ & $19(20,4)$ & $100(32,7)$ & 0,02 & 17 \\
\hline $\begin{array}{l}\text { Unable to walk outside the house without help, } \\
\text { n (\%) }\end{array}$ & $150(37,5)$ & $34(35,1)$ & $116(38,3)$ & 0,56 & 16 \\
\hline Barthel Index, Me (IQR) & $90(70-100)$ & $90(65-97.5)$ & $90(70-100)$ & 0.26 & 6 \\
\hline I-ADL, Me (IQR) & $7(3-11)$ & $7(3-9.25)$ & $8(3-11)$ & 0.09 & 10 \\
\hline Number of chronic diseases, Me (IQR) & $4(3-6)$ & $6(5-7)$ & $4(3-5)$ & $<0.001$ & - \\
\hline Multimorbidity, n (\%) & $201(48.3)$ & $86(87.8)$ & $153(48.1)$ & $<0.001$ & - \\
\hline Number of drugs, Me (IQR) & $7(5-9)$ & $8(6-10)$ & $7(5-9)$ & 0.001 & 9 \\
\hline Polypharmacy, n (\%) & $322(79.1)$ & $86(89.6)$ & $236(75.9)$ & 0.004 & 9 \\
\hline Inactivity, n (\%) & $168(4 \mid .0)$ & $51(52.0)$ & $117(37.5)$ & 0.01 & 8 \\
\hline Hospitalization in the last 12 months, $n(\%)$ & $122(29.5)$ & $37(38.1)$ & $85(26.9)$ & 0.03 & 3 \\
\hline BMI, kg/m², M (SD) & $29.25(5.98)$ & $30,19(5.53)$ & $28,98(6.08)$ & 0.114 & 62 \\
\hline \multicolumn{6}{|l|}{ Medications } \\
\hline B-blockers, n (\%) & $258(63.5)$ & $79(82.3)$ & $179(57.7)$ & $<0.001$ & 10 \\
\hline ACE-Is/ARBs, n (\%) & $259(63.8)$ & $67(69.8)$ & $192(61.9)$ & 0.16 & 10 \\
\hline Calcium channel blockers, $\mathrm{n}(\%)$ & $114(28.1)$ & $22(22.9)$ & $92(29.7)$ & 0.20 & 10 \\
\hline$\alpha$ I-blockers, $\mathrm{n}(\%)$ & $25(6.2)$ & $10(10.4)$ & $15(4.8)$ & 0.05 & 10 \\
\hline Antiarrhythmic, n (\%) & $9(2.2)$ & $6(6.3)$ & $3(1.0)$ & 0.002 & 10 \\
\hline Digoxin, n (\%) & $30(7.4)$ & $26(27.1)$ & $4(1.3)$ & $<0.001$ & 10 \\
\hline Thiazide, n (\%) & $83(20.4)$ & $13(13.5)$ & $70(22.6)$ & 0.06 & 10 \\
\hline Loop diuretics, $n(\%)$ & $100(24.6)$ & $47(49.0)$ & $53(17.1)$ & $<0.001$ & 10 \\
\hline Aldosterone- receptor antagonists, n (\%) & $71(17.5)$ & $32(33.3)$ & $39(12.6)$ & $<0.001$ & 10 \\
\hline Statins, n (\%) & $142(35.0)$ & $29(30.2)$ & $113(36.5)$ & 0.26 & 10 \\
\hline Antiplatelet, n (\%) & $128(31.5)$ & $25(26.0)$ & $103(33.2)$ & 0.19 & 10 \\
\hline Anticoagulants, n (\%) & $64(15.4)$ & $57(58.2)$ & $7(2.2)$ & $<0.001$ & 10 \\
\hline Hemoglobin, mmol/L, M (SD) & $7.76(1.09)$ & $7.64(1.17)$ & $7.80(1.06)$ & 0.19 & 12 \\
\hline Anemia, n (\%) & $177(43.8)$ & $54(56.3)$ & $123(39.9)$ & 0.005 & 12 \\
\hline GFR, $1 / \mathrm{min} / 1.73 \mathrm{~m}^{2}, M(\mathrm{SD})$ & $218(52.4)$ & $51.72(17.73)$ & $60.11(16.31)$ & $<0.001$ & 11 \\
\hline Serum creatinine, mmol/L, Me (IQR) & 86.63 & 99.01 & 84.86 & $<0.001$ & 11 \\
\hline & $(74.26-105.20)$ & $(79.34-119.56)$ & $(72.49-98.12)$ & & \\
\hline HAS-BLED, Me (IQR) & $2(I-2)$ & $2(2-3)$ & $I(I-2)$ & $<0.001$ & 2 \\
\hline HAS-BLED $\geq 3, \mathrm{n}(\%)$ & $63(15.2)$ & $37(38.1)$ & $26(8.2)$ & $<0.001$ & 2 \\
\hline CHADS2-VASC, Me (IQR) & $4(3-5)$ & $5(4-6)$ & $3(3-4.5)$ & $<0.001$ & 2 \\
\hline
\end{tabular}

Notes: ${ }^{a} \chi^{2}$ test or Fisher exact test, as appropriate, for categorical variables. Student's $t$-test or Mann-Whitney test for continuous or interval variables.

Abbreviations: ACEls, angiotensin-converting enzyme inhibitors; AF, atrial fibrillation; ARBs, angiotensin II receptor blockers; BMI, body mass index; GFR, glomerular filtration rate; IADL, instrumental activities of daily living; IQR, interquartile range; $M$, mean value; Me, median value; $n$, number of cases.

BMI value in the control group $\left(28.98 \pm 6.08 \mathrm{~kg} / \mathrm{m}^{2}\right.$, $P=0.44)$.

The thromboembolic risk evaluated with CHA2DS2VASc scale was generally high in both groups; however, in patients with AF, the median score was significantly higher (Me 5; IQR-4-6 vs Me 3; IQR-3-4.5, $P<0.001$ ). The risk of bleeding assessed with HAS-BLED scale was also higher in patients with AF comparing to the control group. The hemoglobin level was similar in both groups, but anemia was significantly more often diagnosed within AF group (56.3\% vs $39.9 \%, P=0.005)$.

The AF+ and AF- groups did not differ in place of residence, education, inability to walk outside the house, the Barthel Index and IADL-scale scores, BMI, obesity, 
percentages of patients using ACE-Is or ARBs, or thiazides, statins, antiplatelet drugs, prevalence of coronary artery disease, myocardial infarction, hypertension, COPD, thyroid dysfunction, smoking, and alcohol drinking.

Our analyses revealed possible risk factors and determinants for AF (Table 2). The most significant factors were congestive heart failure (risk ratio, 4.12; 95\% CI, 2.8-6.1; $P<0.001$ ), peripheral arterial disease (risk ratio, 2.09; 95\% CI, 1.5-3.0; $P<0.001$ ); age $75+$ years (risk ratio, 3.51; $95 \%$ CI, $1.5-8.3 ; P=0.001$ ), history of stroke or TIA (risk ratio, $1.86 ; 95 \% \mathrm{CI}, 1.3-2.7 ; P=0.003$ ), and chronic kidney disease (risk ratio, $1.71 ; 95 \% \mathrm{CI}, 1.2-2.5 ; P=0.003$ ). Male patients presented a 1.59-fold higher risk of AF (95\% CI, 1.1-2.3; $P=0.01)$, and patients with diabetes a 1.46 -fold higher risk (95\% CI, 1.0-2.1; $P=0.04$ ). The risk of AF was 1.56-fold higher $(95 \% \mathrm{CI}, 1.1-2.2 ; P=0.01)$ if the patient was physically inactive. No significant increase in the risk of AF was observed for such recognized risk factors as coronary artery disease, myocardial infarction, hypertension, COPD, thyroid dysfunction, obesity, smoking, and alcohol drinking.

In multivariable logistic regression analysis, an independent effect associated with the diagnosis of AF was observed among patients with the congestive heart failure (odds ratio, 5.42; 95\% CI, 3.13-9.3; $P<0.001)$, in the age of 75 years or older (odds ratio, $4.18 ; 95 \% \mathrm{CI}, 1.49-11.7 ; P=0.007$ ), or with the history of stroke or TIA (odds ratio, 2.15; 95\% CI, $1.09-4.2 ; P=0.03$ ), when controlling for peripheral arterial disease, diabetes, myocardial infarction, chronic kidney disease, and gender (Table 3). We did not include CHA2DS2VASc scale score in the model (as it was connected with the significant multicollinearity effect), and inactivity (as it should be treated as the result of AF and its co-morbidity rather in our study population, then the risk factor for $\mathrm{AF}$ ).

ROC curve analysis was performed to test the predictive discrimination of patients with and without AF with the identified significant predictors of AF and CHA2DS2VASc scale (Figure 1 and Table 4). The largest ROC area of 0.75 was obtained for CHA2DS2-VASc score, and we found the value 4.0 as the best cut-off value for CHA2DS2-VASc; it yielded the best combination of sensitivity (88.7\%; 95\% CI: 80.6-94.2) and specificity (50.5\%; 95\% CI: 44.8-56.1) for prediction of AF. The next largest ROC area of 0.72 was obtained for CHF, and it did not differ significantly from that for the CHA2DS2-VASc scale (difference between areas- 0.03 $\pm 0.02, P=0.19$ ), but sensitivity of CHF was noticeably lower (72.4\%; 95\% CI: 62.5-81.0).

\section{Discussion}

A "growing epidemic" of AF is observed worldwide due to the aging of the populations, better survival in some diseases, and better detection of arrhythmias. ${ }^{17}$ Our study has confirmed that AF is a very common arrhythmia among geriatric ward patients; it was found in $23 \%$ of

Table 2 Clinical correlates and risk factors of AF in the study group

\begin{tabular}{|c|c|c|c|c|c|}
\hline Parameter & Total & AF+ group & AF- group & $\operatorname{RR}(95 \% \mathrm{CI})$ & $P$-values \\
\hline No. (\%) of patients & $416(100.0)$ & $98(23.6)$ & $318(76.4)$ & & \\
\hline Age, 75+, n (\%) & $350(84.1)$ & $93(94.9)$ & $257(80.8)$ & $3.51(1.5-8.3)$ & 0.001 \\
\hline Gender, men, n (\%) & $94(22.09)$ & $31(31.6)$ & $63(19.8)$ & $1.59(1.1-2.3)$ & 0.01 \\
\hline Coronary artery disease, $\mathrm{n}(\%)$ & $223(53.6)$ & $58(59.2)$ & $165(51.9)$ & $1.26(0.9-1.8)$ & 0.21 \\
\hline Myocardial infarction, n (\%) & $39(9.4)$ & $14(14.3)$ & $25(7.9)$ & $1.61(1.0-2.6)$ & 0.06 \\
\hline Hypertension, n (\%) & $327(78.6)$ & $80(81.6)$ & $247(77.7)$ & $1.21(0.8-1.9)$ & 0.40 \\
\hline Peripheral arterial disease, $\mathrm{n}(\%)$ & $64(15.4)$ & $27(27.6)$ & $37($ (II.6) & $2.09(1.5-3.0)$ & $<0.001$ \\
\hline CHF, n (\%) & $162(38.9)$ & 71 (72.4) & 91 (28.6) & $4.12(2.8-6.1)$ & $<0.001$ \\
\hline Stroke and TIA, n (\%) & $56(13.5)$ & $22(22.4)$ & $34(10.7)$ & $1.86(1.3-2.7)$ & 0.003 \\
\hline Diabetes, n (\%) & $126(30.3)$ & $38(38.8)$ & $88(27.7)$ & $1.46(1.0-2.1)$ & 0.04 \\
\hline COPD, n (\%) & $42(10,1)$ & $13(13,3)$ & $29(9,1)$ & $1.4(0.8-2.2)$ & 0,23 \\
\hline Thyroid dysfunction, n (\%) & $74(17,8)$ & $17(17,4)$ & $57(17,9)$ & $0.97(0.6-1.5)$ & 0,89 \\
\hline Chronic kidney disease, n (\%) & $218(52.4)$ & $64(65.3)$ & $154(48.4)$ & I,7I (I.2-2.5) & 0.003 \\
\hline $\mathrm{BMI} \geq 30 \mathrm{~kg} / \mathrm{m}^{2}, \mathrm{n}(\%)$ & I $48(4 \mid .8)$ & $36(45.6)$ & $112(40.7)$ & $1.17(0.8-1.7)$ & 0.44 \\
\hline Smoking, n (\%) & $35(8.8)$ & $10(10.8)$ & $25(8.2)$ & $1.25(0.7-2.2)$ & 0.45 \\
\hline Alcohol drinking, $n(\%)$ & $7(1.8)$ & $3(3.2)$ & $4(1.4)$ & $1.82(0.8-4.4)$ & 0.24 \\
\hline Inactivity ${ }^{\mathrm{d}}, \mathrm{n}(\%)$ & $168(41.0)$ & $5 I(52.0)$ & 117 (37.5) & $1.56(1.1-2.2)$ & 0.01 \\
\hline
\end{tabular}

Note: ${ }^{a} \chi^{2}$ test.

Abbreviations: $\mathrm{AF}$, atrial fibrillation; $\mathrm{BMI}$, body mass index; $\mathrm{CHF}$, congestive heart failure; $\mathrm{Cl}$, confidence interval; $\mathrm{COPD}$, chronic obstructive pulmonary disease; $\mathrm{n}$, number of cases; RR, relative risk; TIA, transient ischemic attack. 
Table 3 Risk factors associated with AF - multivariable logistic regression model

\begin{tabular}{|l|l|l|l|}
\hline & OR & 95\% CI & P-value \\
\hline Congestive heart failure & 5.43 & $3.14-9.39$ & $<0.00 \mathrm{I}$ \\
Age, 75+ years & 4.00 & $1.43-11.20$ & 0.008 \\
Stroke and TIA & 2.10 & $1.06-4.13$ & 0.03 \\
Gender, men & 1.50 & $0.82-2.73$ & 0.19 \\
Peripheral arterial disease & 1.50 & $0.76-2.95$ & 0.24 \\
Diabetes & 1.38 & $0.80-2.37$ & 0.25 \\
Myocardial infarction & 0.68 & $0.31-1.52$ & 0.35 \\
Chronic kidney disease & 1.17 & $0.68-2.02$ & 0.58 \\
\hline
\end{tabular}

Abbreviations: $\mathrm{Cl}$, confidence interval; OR, odds ratio; TIA, transient ischemic attack.

the group studied. It was more frequent in the population we examined than in the general population of older people, which resulted probably from the more frequent accumulation of recognized risk factors for AF in this group. ${ }^{18}$ The majority of our patients were 75-year-old or older, very frequently burdened with hypertension, coronary artery disease, congestive heart failure, history of stroke or TIA, diabetes, and peripheral artery disease.

The diagnosis of AF is often delayed. It is diagnosed more often when patients start to have symptoms and seek medical evaluation or incidentally, when they attend a health care setting for another reason. A high percentage of older patients diagnosed as having $\mathrm{AF}$ in screening studies had no attributable symptoms and were not aware to have AF. ${ }^{19}$ Therefore, a clear need exists for an improvement in AF detection and diagnosis. This task is delegated primarily to the primary health care. Unfortunately, this can be difficult to perform in daily clinical practice due to the high prevalence of cognitive and functional impairment among patients in older age. Furthermore, it is complicated by paroxysmal or asymptomatic character of arrhythmia - socalled "silent AF". ${ }^{20}$ A detailed screening is not always possible in all patients of the primary health care settings. The obstacles indicated include also organizational barriers, like the lack of time, staff, and capacity. $^{21}$ It is still not decided when, or how often, ECG/Holter ECG in the high-risk population should be done.

In our research, the most important predictor of $\mathrm{AF}$ was congestive heart failure (CHF). The ROC evaluation showed that of three single significant predictors of AF (the other two were advanced old age and previous history of stroke or TIA) only CHF showed a predictive value with satisfactory AUC. The relationship between AF and CHF can be bi-directional - CHF contributes to AF, but AF can also be a cause of exacerbation of CHF symptoms. For screening purpose, however, determining the direction of interaction is not so important. Advanced old age and

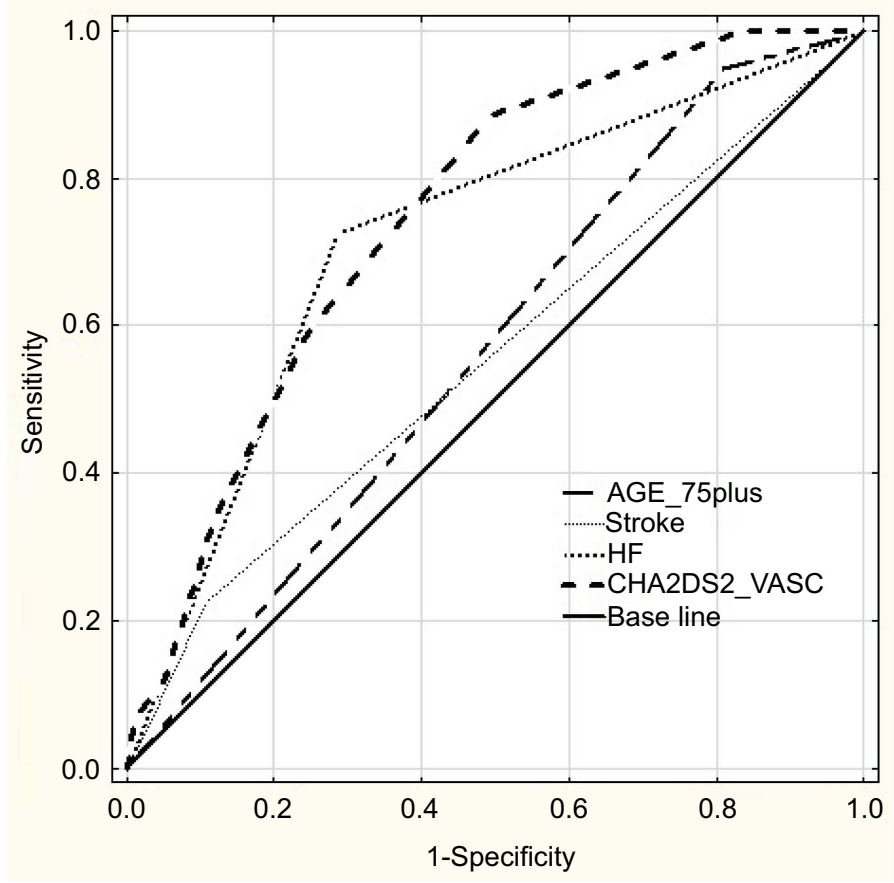

Figure I Receiver operating characteristics (ROC) curve analysis for the ability of age 75 or above (AGE_75plus), history of stroke (STROKE), heart failure (HF), and CHA2DS2-VASC score (CHA2DS2_VASC) to predict atrial fibrillation (AF). 
Table 4 The values of CHA2DS2-VASC score, CHF diagnosis, history of stroke/TIA, and age 75years or above for the prediction of $\mathrm{AF}$ and their overall diagnostic effectiveness

\begin{tabular}{|l|l|l|l|l|}
\hline ROC Index & CHA2DS2-VASC score & CHF & Age, 75+years & Stroke-TIA \\
\hline AUC & 0.75 & 0.72 & 0.57 & 0.56 \\
$95 \%$ Cl of AUC & $0.70-0.80$ & $0.66-0.78$ & $0.51-0.63$ & $0.49-0.63$ \\
P-value & $<0.00 I$ & $<0.001$ & 0.02 & 0.09 \\
Youden Index J & 0.391 & 0.438 & 0.141 & 0.118 \\
Cut-off criterion & 4 & $I^{*}$ & $I^{*}$ & 0.22 \\
Sensitivity (\%) & 0.89 & 0.72 & 0.95 & $0.15-0.32$ \\
$95 \%$ Cl of sensitivity & $0.81-0.94$ & $0.63-0.81$ & $0.89-0.98$ & 0.89 \\
Specificity & 0.51 & 0.71 & 0.19 & $0.85-0.93$ \\
$95 \%$ Cl of specificity & $0.45-0.56$ & $0.66-0.76$ & $0.15-0.24$ & 1.17 \\
Positive likelihood ratio & 1.79 & 2.53 & 2.1 & 0.27 \\
Negative likelihood ratio & 0.23 & 0.39 & 0.87 & \\
\hline
\end{tabular}

Note: $* I$ means that characteristic is present (opposite to 0 - absent).

Abbreviations: AF, atrial fibrillation; AUC, under individual roc curves, $\mathrm{CHF}$, congestive heart failure; $\mathrm{Cl}$, confidence interval; ROC, receiver operator characteristic; TIA, transient ischemic attack.

previous history of stroke or TIA have been generally approved as the most important thromboembolic risk factors, and that was emphasized in the CHA2DS2-VASc scale - the assessed patient receives 2 points in case of each of them. ${ }^{9}$

Considering the fact that variables correlating with AF arrhythmia are also important thromboembolic risk factors, we tried to evaluate the predictive value of the CHA2DS2-VASc scale as the whole. In the ROC analysis, the result obtained in this scale had the highest predictive ability, with the suggested cut-off being the score equal to or higher than 4 points. CHA2DS2-VASc scale score was confirmed to have a good predictive value in the prediction of new-onset AF occurrence, and the risk increased with increasing score points. $^{22}$ Other, recently carried out research showed that this scale can be applied to stroke prediction in persons without $\mathrm{AF}^{23}$ It is also useful in anticipating cardiovascular/cerebrovascular incidents occurrence in coronary artery disease patients without $\mathrm{AF}^{24}$ Considering the results of our research, it is worth to consider its use for the purpose of screening of AF.

Although the ROC analysis revealed that both CHA2DS2-VASc and congestive heart failure diagnosis score can be used as predictive markers for $\mathrm{AF}$ in the studied group (AUC for both predictors did not differ significantly), the sensitivity of the value of 4 or more in was noticeable higher than of CHF (88.7\% versus $72.4 \%$ ). It is important from the point of view of the effect we would like to achieve as a result of screening. We should also remember that a significant part of CHF in older people is unrecognized. ${ }^{25,26}$ Echocardiography is not readily available in primary care, and particularly recognizing HF in the early stage is challenging. This largely limits the use of this variable as a reliable, single predictor of AF for screening purposes in primary care. CHA2DS2VASc scale includes some variables, for which there are no diagnostic doubts (such as age, gender), and it could allow for a more precise selection of the population, which should be covered with AF screening at first.

However, our study has got some limitations that should be considered. First of all, the evaluated group of patients was not a randomly selected sample. So the results must be treated with caution, as predictive abilities of analyzed variables, including CHA2DS2-VASc score, for the general population can differ. Some limitations result from partially retrospective character of the study conducted. As a result, we could not include all of the recognized AF risk factors listed in the guidelines (such as obstructive sleep apnea or valvular heart diseases) in our analysis. Information about their prevalence was not generally incorporated in patients' medical records. Another consequence of retrospective approach was also missing values in some variables (showed in the tables). It should be also mentioned that the diagnosis of AF was based mainly on ECG, and only in some cases on $24 \mathrm{hr}$ ECG monitoring. Not every patient without AF in standard ECG was monitored with Holter ECG. It could possibly result in not recognizing all of the "silent" AF cases in hospitalized patients. In another study performed in the geriatric department in Saint Jan Hospital Bruges, AF screening leads to an overall prevalence of $46 \%$ in hospitalized patients $(33 \%$ patients with AF identified by routine clinical care and 13\% with daily short-term rhythm strip 
recordings added to it). ${ }^{27}$ Diagnosis of AF comorbidities based on patients' medical records could also result in not recognizing all of their cases.

\section{Conclusions}

In summary, we showed that AF is a common rhythm disorder in older age and can affect up to $25 \%$ of geriatric inpatients. Congestive heart failure, history of stroke or transient ischemic attack, and age 75 years or above are the main independent predictors of AF in patients hospitalized in the geriatric ward. The use of CHA2DS2-VASc scale could allow to discriminate between individuals with and without AF with the value 4 or more as the best cutoff point, and it can be a first-step screening instrument.

\section{Acknowledgments}

This work was supported in part by funds of the Medical University in Bialystok research project no N/ST/ZB/15/ 001/3301 (granted to ZBW) and N/ST/MN/18/001/3301 (granted to MLS).

\section{Author contributions}

All authors contributed to data analysis, drafting and revising the article, gave final approval of the version to be published, and agree to be accountable for all aspects of the work.

\section{Disclosure}

The authors report no conflicts of interest in this work.

\section{References}

1. Haim M, Hoshen M, Reges O, Rabi Y, Balicer R, Leibowitz M. Prospective national study of the prevalence, incidence, management and outcome of a large contemporary cohort of patients with incident non-valvular atrial fibrillation. J Am Heart Assoc. 2015;4(1):e001486. doi:10.1161/JAHA.114.001486

2. Vanbeselaere V, Truyers C, Elli S, et al. Association between atrial fibrillation, anticoagulation, risk of cerebrovascular events and multimorbidity in general practice: a registry-based study. BMC Cardiovasc Disord. 2016;16:61. doi:10.1186/s12872-016-0371-7

3. Andersson T, Magnuson A, Bryngelsson IL, et al. All-cause mortality in 272,186 patients hospitalized with incident atrial fibrillation 1995-2008: a Swedish nationwide long-term case-control study. Eur Heart J. 2013;34(14):1061-1067. doi:10.1093/eurheartj/ehs469

4. Benjamin EJ, Wolf PA, D‘Agostino RB, Silbershatz H, Kannel WB, Levy D. Impact of atrial fibrillation on the risk of death: the Framingham Heart Study. Circulation. 1998;98(10):946-952.

5. Goren A, Liu X, Gupta S, Simon TA, Phatak H. Quality of life, activity impairment, and healthcare resource utilization associated with atrial fibrillation in the US National Health and Wellness Survey. PLoS One. 2013;8(8):e71264. doi:10.1371/journal.pone.0071264
6. Marzona I, O`Donnell M, Teo K, et al. Increased risk of cognitive and functional decline in patients with atrial fibrillation: results of the ONTARGET and TRANSCEND studies. Cmaj. 2012;184(6):E329E336. doi:10.1503/cmaj.111173

7. von Eisenhart Rothe A, Hutt F, Baumert J, et al. Depressed mood amplifies heart-related symptoms in persistent and paroxysmal atrial fibrillation patients: a longitudinal analysis-data from the German Competence Network on Atrial Fibrillation. Europace. 2015;17 (9):1354-1362. doi:10.1093/europace/euv018

8. Alnsasra H, Haim M, Senderey AB, et al. Net clinical benefit of anticoagulant treatments in elderly patients with nonvalvular atrial fibrillation: experience from the real world. Heart Rhythm. 2019;16 (1):31-37. doi:10.1016/j.hrthm.2018.08.016

9. Kirchhof P, Benussi S, Kotecha D, et al. 2016 ESC guidelines for the management of atrial fibrillation developed in collaboration with EACTS. Rev Esp Cardiol (Engl Ed). 2017;70(1):50.

10. Wojszel ZB. Geriatric Syndromes and Care Services in Late Old Age. Multidimensional Analysis on the Example of Chosen Environments of the Podlasie Region. Bialystok: Wydawnictwo Uniwersyteckie Trans Humana; 2009.

11. Zungsontiporn N, Link MS. Newer technologies for detection of atrial fibrillation. BMJ. 2018;363:k3946. doi:10.1136/bmj.k4029

12. Zoni-Berisso M, Filippi A, Landolina M, et al. Frequency, patient characteristics, treatment strategies, and resource usage of atrial fibrillation (from the Italian Survey of Atrial Fibrillation Management [ISAF] study). Am J Cardiol. 2013;111(5):705-711. doi:10.1016/j.amjcard.2012.11.026

13. Proietti M, Mairesse GH, Goethals P, et al. A population screening programme for atrial fibrillation: a report from the Belgian Heart Rhythm Week screening programme. Europace. 2016;18 (12):1779-1786. doi:10.1093/europace/euw069

14. Mahoney FI, Barthel DW. Functional evaluation: the barthel index. Md State Med J. 1965;14:61-65.

15. Fillenbaum GG, Smyer MA. The development, validity, and reliability of the OARS multidimensional functional assessment questionnaire. J Gerontol. 1981;36(4):428-434.

16. Rodjer L, Jonsdottir IH, Rosengren A, et al. Self-reported leisure time physical activity: a useful assessment tool in everyday health care. BMC Public Health. 2012;12:693. doi:10.1186/1471-2458-12-693

17. Chugh SS, Havmoeller R, Narayanan K, et al. Worldwide epidemiology of atrial fibrillation: a Global Burden of Disease 2010 Study. Circulation. 2014;129(8):837-847. doi:10.1161/CIRCULATIONAHA.113.005119

18. Langenberg M, Hellemons BS, van Ree JW, et al. Atrial fibrillation in elderly patients: prevalence and comorbidity in general practice. BMJ. 1996;313(7071):1534. doi:10.1136/bmj.313.7071.1534

19. Frewen J, Finucane C, Cronin H, et al. Factors that influence awareness and treatment of atrial fibrillation in older adults. QJM. 2013;106(5):415-424. doi:10.1093/qjmed/hct060

20. Verbiest-van Gurp N, van Bladel PJM, van Kesteren HAM, Erkens PM, Stoffers H. Current practice of Dutch cardiologists in detecting and diagnosing atrial fibrillation: results of an online case vignette study. Neth Heart J. 2017;25(10):567-573. doi:10.1007/s12471-017-1010-3

21. Chiang CE, Naditch-Brule L, Brette S, et al. Atrial fibrillation management strategies in routine clinical practice: insights from the International RealiseAF Survey. PLoS One. 2016;11(1):e147536. doi:10.1371/journal.pone. 0147536

22. Saliba W, Gronich N, Barnett-Griness O, Rennert G. Usefulness of CHADS2 and CHA2DS2-VASc scores in the prediction of new-onset atrial fibrillation: a population-based study. Am J Med. 2016;129 (8):843-849. doi:10.1016/j.amjmed.2016.02.029

23. Liu FD, Shen XL, Zhao R, et al. Predictive role of CHADS2 and CHA2DS2-VASc scores on stroke and thromboembolism in patients without atrial fibrillation: a meta-analysis. Ann Med. 2016;48 (5):367-375. doi:10.1080/07853890.2016.1179390 
24. Tabata N, Yamamoto E, Hokimoto S, et al. Prognostic value of the CHADS2 score for adverse cardiovascular events in coronary artery disease patients without atrial fibrillation-A multi-center observational cohort study. J Am Heart Assoc. 2017;6(8). doi:10.1161/JAHA.117.006355

25. van Riet EE, Hoes AW, Limburg A, Landman MA, van der Hoeven H, Rutten FH. Prevalence of unrecognized heart failure in older persons with shortness of breath on exertion. Eur J Heart Fail. 2014;16(7):772-777. doi:10.1002/ejhf.110
26. Barrios V, Escobar C, De La Sierra A, Llisterri JL, Gonzalez-Segura D. Detection of unrecognized clinical heart failure in elderly hypertensive women attended in primary care setting. Blood Press. 2010;19(5):301-307. doi:10.3109/08037051.2010.488051

27. Tavernier R, Wolf M, Kataria V, et al. Screening for atrial fibrillation in hospitalised geriatric patients. Heart. 2018;104(7):588-593. doi:10.1136/heartjnl-2017-311981

\section{Publish your work in this journal}

Clinical Interventions in Aging is an international, peer-reviewed journal focusing on evidence-based reports on the value or lack thereof of treatments intended to prevent or delay the onset of maladaptive correlates of aging in human beings. This journal is indexed on PubMed Central, MedLine, CAS, Scopus and the Elsevier
Bibliographic databases. The manuscript management system is completely online and includes a very quick and fair peer-review system, which is all easy to use. Visit http://www.dovepress.com/ testimonials.php to read real quotes from published authors. 